\title{
Development of a Multi-Plex Electrochemiluminescent Assay for the Detection of Serum Antibody Responses to Meningococcal Conjugate Vaccines
}

\author{
Lani Indrawati*, Jon H. Heinrichs, Emily P. Wen, Julie M. Skinner \\ Vaccines Research, Merck Research Laboratories, West Point, New York, USA. \\ Email: *lani_indrawati@merck.com \\ Received November $10^{\text {th }}, 2011$; revised December $20^{\text {th }}, 2011$; accepted January $15^{\text {th }}, 2012$
}

\begin{abstract}
Neisseria meningitidis is a gram negative diplococcal bacterium. Worldwide, $N$. meningitidis is the leading cause of bacterial meningitis and sepsis, with five serogroups (A, B, C, Y, and W-135) responsible for the majority of the disease. Multivalent (A, C, Y, and W-135) polysaccharide and conjugate vaccines have been licensed in the United States and elsewhere and are widely available. We have developed a multi-plexed electrochemiluminescent assay to quantitate serum antibody responses to meningococcal polysaccharides A, C, Y, and W-135 to allow for rapid evaluation of licensed and investigational vaccines. A 96-well plate containing a carbon electrode arrayed with polysaccharides A, C, Y, and W-135 on separate spots within each well has been developed for simultaneous detection of polysaccharidespecific antibodies in serum samples from vaccinated individuals. The assay conditions were optimized using the antimeningococcal serogroup A/C reference serum pool, CDC 1992 (NIBSC 99/706), through evaluation of plate types, coating polysaccharide concentrations, and blocking and serum diluent buffers. Comparison of single and multi-plex assays demonstrated the sensitivity, specificity, and speed of the multi-plex format for the quantification of serum antibody responses to $N$. meningitidis polysaccharides A, C, Y and W-135.
\end{abstract}

Keywords: Neisseria meningitidis; Electrochemiluminescent Detection Serum Antibody

\section{Introduction}

Infections caused by the bacterium Neisseria meningitisdis remain an important medical need, particularly in the developing world where periodic outbreaks are responsible for thousands of deaths [1]. Despite the availability of effective antibiotics, death occurs in approximately $10 \%$ of cases, and up to $20 \%$ of survivors are afflicted with permanent disabilities including limb amputations, deafness and other serious sequelae [2]. Although 13 serogroups of meningococci have been identified based upon the structural identity of their polysaccharide capsules, the vast majority of disease is caused by only five groups (A, B, C, Y, and W-135) [3]. Recently, efficacious vaccines targeting four of these serogroups (A, C, Y and W135) have been developed by direct conjugation of polysaccharide antigens to protein carriers [3-7]. These vaccines provide benefit over traditional, unconjugated polysaccharide (Ps) vaccines by inducing T-cell memory, and afford long-term immunity in both children and adults [2].

Licensure of meningococcal vaccines typically relies

${ }^{*}$ Corresponding author. on the demonstration of immunological correlates of protection as disease incidence in the developed world is extremely low. In particular, the demonstration of functional antibody response is required and typically demonstrated using the serum bactericidal assay (SBA). Total serum-antibody specific for each of the Ps components of the vaccine under evaluation are usually assessed by enzyme-linked immunosorbent assay (ELISA). Evaluating antibody concentrations via ELISA is both time consuming and serum consuming. To create a higher throughput assay, we have developed an electrochemiluminescent (ECL)-based multi-plex assay for the quantitation of antibody responses to meningococcal polysaccharide from serogroups A, C, Y, and W-135.

Compared to traditional ELISA methods, ECL-based assays are highly sensitive, reproducible, robust, faster, and require lower sample volumes [8,9]. Recently, several groups have used a fluorescent bead based approach to develop multi-plex assays to analyze antibody responses to $N$. meningitidis [10,11]. However, limitations remain for this type of assay, including a requirement for covalent conjugation of polysaccharides to beads, which 
may impact polysaccharide conformation, and reliance on fluidic based measurement techniques which are intrinsically slow and prone to clogging. As an alternative, we have developed an assay based upon technology developed by Meso-Scale Discovery (MSD, Gaithersburg, MD) which allowed us to compare single vs. multi-plex assays in a 96-well plate format. The MSD Multi-array ${ }^{\circledR}$ and Multi-spot ${ }^{\circledR}$ 96-well plates have carbon coated wells with electrodes that produce chemical energy when subjected to an electrical charge (www.mesoscale.com). When combined with a MSD SULFO-TAG ${ }^{\circledR}$ antibody, the chemical energy is transformed to emitted light which is measured using a high-resolution CCD camera. Serum antibody concentrations are determined by comparison to a standard curve generated using a pooled human antibody preparation (NIBSC 99/706). A similar assay was developed by Marchese et al. to measure antibodies in human serum for eight of the serotypes contained in the Streptococcus pneumoniae vaccine PNEUMOVAX $^{\circledR} 23$ [12]. Herein we describe the development of a high throughput, multi-plexed ECL assay to simultaneously evaluate antibody responses to $N$. meningitidis serogroup Ps (MnPs) A, C, Y, and W-135.

\section{Materials and Methods}

\subsection{N. meningitidis Polysaccharides (MnPs)}

Serogroups A, C, Y, and W-135 MnPs used in this study were isolated separately from the native organisms at Merck Research Labs, West Point, PA, using modified procedures described previously $[13,14]$. Each purified MnPs was reconstituted in sterile pyrogen-free water to a final concentration of $1 \mathrm{mg} / \mathrm{ml}$. These MnPs aliquots were placed at $-70^{\circ} \mathrm{C}$ for long term storage or at $4^{\circ} \mathrm{C}$ for up to one month.

\subsection{Meningococcal Reference Sera}

The anti-meningococcal serogroup $\mathrm{A} / \mathrm{C}$ reference serum pool, CDC 1992 (NIBSC; code: 99/706), is a pool of sera from 20 healthy adults following a single immunization of meningococcal polysaccharide vaccine $(50 \mu \mathrm{g}$ of A, C, $\mathrm{W}-135$, and Y polysaccharide/0.5 ml; Menomune; Connaught Laboratories, Inc., Swiftwater, PA.) [15]. The US FDA Pneumococcal reference standard, lot 89SF-2 (Lederle-Praxis Biologicals), was prepared by pooling 17high titered sera from adults following immunization with Pnu-Imune (a 23-valent Pneumococcal vaccine; Lederle), Menomune and ProHIBIT (a $\mathrm{H}$. influenzae conjugate vaccine; Connaught) [16,17].

\subsection{Meningococcal Polysaccharide ECL Assay}

Each polysaccharide was coated on the carbon surface at either $50 \mathrm{ng} /$ well (single-plex) or $10 \mathrm{ng} / \mathrm{spot}$ (four-plex). Uncoated reactive sites on the carbon surface were blocked by addition of a blocking solution and incubation at room temperature for one hour. Plates were washed with PBS $+0.05 \%$ Tween-20 (PBST) and $25 \mu \mathrm{l}$ test serum samples pre-diluted in specified diluent buffer were then added to each well. Following incubation for 1 hour on a shaker $(\sim 550 \mathrm{rpm})$ at room temperature and subsequent washing with PBST, $25 \mu \mathrm{l} /$ well of Sulfo-tag labeled goat anti-human IgG secondary antibody (MSD) was added and the plates were incubated at room temperature for 1 hour on a shaker. The plates were again washed with PBST and $150 \mu \mathrm{l} /$ well of 1x Read Buffer-T (with surfactant, MSD) diluted in distilled $\mathrm{H}_{2} \mathrm{O}$ was added. The plates were analyzed on a Sector Imager 6000 (SI-6000) instrument with MSD Workbench ${ }^{\mathrm{TM}}$ v.3 software.

\subsection{ECL Assay Optimization}

Assay optimization was performed in the single-plex format with the CDC 1992 control reference serum. The following parameters were evaluated: 1) comparison of high binding and standard binding plates; 2) evaluation of blocking solutions (5\% MSD Blocker A, 5\% fetal bovine serum/FBS, 3\% MSD Blocker B, and 5\% MSD Blocker A + 0.5\% MSD Blocker B); 3) effect of serum diluent buffer (PBS, PBS $+1 \%$ MSD Blocker $\mathrm{B} \pm$ the addition of various concentrations of $\mathrm{NaCl}$ ). The optimal assay conditions determined in the single-plex format were then applied for multi-plex assay development.

\subsection{Specificity of the Multi-Plex ECL Assay}

CDC 1992 and 89SF-2 reference sera were diluted 1:100 in PBS $+1 \%$ MSD Blocker B. The sera were then divided into five aliquots and each aliquot was incubated for one hour with $10 \mu \mathrm{g} / \mathrm{ml}$ MnPs A or C or Y or W-135 or serum diluent buffer only. The percent inhibition was calculated using the formula [1 - ((serum concentration in the presence of inhibitor/(serum concentration in the absence of inhibitor)) $] \times 100 \%$.

\subsection{Meningococcal Vaccine Immunogenicity in Infant Rhesus Monkeys}

Two infant rhesus monkey (IRM) studies were performed at Covance, Inc. (Alice, TX) to assess the immunogenicity of commercially available meningococcal vaccines. These infant rhesus monkeys were 2 - 3 months old at the initiation of the studies. In each study, vaccines were given at half the human dose. In the first study, IRMs (five IRMs/group) were immunized with Menomune $^{\mathbb{R}}(25 \mu \mathrm{g} \mathrm{A} / \mathrm{C} / \mathrm{Y} / \mathrm{W}-135$ meningococcal polysaccha- 
ride vaccine, Sanofi Pasteur, Swiftwater, PA). IRMs were vaccinated three times (days 0,28 , and 168) and serum samples were collected prior to the first vaccine dose (day 0), post-dose 1 (day 28), post-dose 2 (day 56), and post-dose 3 (day 210). In the second study, IRMs (eight IRMs/group) were immunized with Menactra ${ }^{\circledR}$ (2 $\mu \mathrm{g}$ each of Ps A, C, Y, and W-135 conjugated to Diphtheria toxoid protein, Sanofi Pasteur, Swiftwater, PA). Three immunizations were also delivered in this study (days 0,56 , and 112). Sera were collected on day 0, postdose 1 (day 28), post-dose 2 (day 84), and post-dose 3 (day 140). All studies were conducted under the auspices of the Institutional Animal Care and Use Committee of both Merck Research Labs and Covance, Inc. These serum samples were tested in the multi-plex ECL assay. The IgG concentration in each serum was calculated using Workbench ${ }^{\mathrm{TM}}$ v.3 software using CDC 1992 reference as assay standard.

\subsection{Statistical Methodology}

All graphs and statistical analysis were done with GraphPad Prism (version 5) software. Analysis was completed using the log transformed mean ECL units. The effect of various serum diluent buffers in reducing the level of background signal was assessed by comparing the mean ECL signals between wells containing no antigen versus wells with MnPs. This was analyzed using a two sided ttest with $95 \%$ confidence interval.

\section{Results}

\subsection{Development and Optimization of the Single-Plex ECL Assay}

Initial studies evaluated the relative contribution of standard vs. high binding Multi-array ${ }^{\circledR}$ plates to the signal to noise ratio of the assay. It was determined that the CDC 1992 reference serum produced a higher signal to noise ratio on the high binding than on the standard binding plate (data not shown). The impact of various coating concentrations $(5,50$, and $250 \mathrm{ng} / \mathrm{well})$ of MnPs A, C, Y, and $\mathrm{W}-135$ were then evaluated and it was determined that $50 \mathrm{ng} / \mathrm{well}$ of MnPs was optimal for the single-plex assay. Due to poor adsorption of the negatively charged Ps to polystyrene microtiter plates, traditional ELISA assays require co-incubation of MnPs with methylated human serum albumin (mHSA) to be used as coating antigen $[18,19]$. We examined the impact of mHSA on binding of MnPs A to the carbon ECL plates. Binding of this Ps to the ECL plates was essentially equivalent with or without the addition of mHSA (data not shown). Additionally, the signal levels produced by the CDC 1992 reference serum were similar in the presence or absence of mHSA (data not shown). This demonstrated that MnPs could be absorbed directly by the carbon surface inside of the wells.

We also examined several blocking solutions to determine their effect on reducing the level of non-specific signal in the ECL assay. The blocking solutions evaluated were 5\% MSD Blocker A (BSA), 5\% FBS, 3\% MSD Blocker B (non-fat milk), and a combination of 5\% MSD Blocker A $+0.5 \%$ MSD Blocker B. The use of 3\% MSD Blocker $\mathrm{B}$ a blocking solution resulted in the highest signal/noise ratio. Therefore, it was subsequently selected as the blocking solution for the ECL assay (data not shown).

We next evaluated the contribution of various serum diluent buffers including, PBS, PBS $+1 \%$ Blocker B, and PBS $+1 \%$ Blocker B supplemented with either 50, 100,250 , or $500 \mathrm{mM}$ of $\mathrm{NaCl}$, to improve the assay performance (Figure 1). High non-specific binding to un-coated wells was observed when PBS was used to dilute the CDC 1992 reference serum (Figure 1(a)). The level of serum non-specific binding was reduced significantly just by adding $1 \%$ Blocker B to PBS $(\mathrm{P}<0.0001)$ (Figure 1(b)). Adding $\mathrm{NaCl}$ to this diluent buffer could reduce the level of non-specific binding at the lower end of the serum dilution (1:100 - 1:400). However, increasing $\mathrm{NaCl}$ concentrations also lowered the specific antibody signals and significantly $(\mathrm{P}<0.0001)$ affected the response detected to MnPs A (Figures 1(c)-(f)).

\subsection{Development and Optimization of Multi-Plex ECL Assay Units}

The assay conditions determined as optimal for the single-plex format were then used to bridge this assay to a multi-plex format. To confirm selection of the plate used for the multi-plex assay, we compared standard and high binding Multi-plex ${ }^{\circledR}$ plates (Figure 2). Titration of CDC 1992 reference serum on both types of Multi-plex ${ }^{\circledR}$ plates revealed similar low levels of non-specific antibody binding to uncoated wells. However, unlike the result obtained in the single-plex format, the reference serum demonstrated higher signal levels for all four MnPs on the standard compared to the high binding plate. Subsequent development of the multi-plex assay utilized standard binding plates.

\subsection{Specificity of Multi-Plex ECL Assay}

The specificity of the multi-plex ECL assay was assessed through competitive inhibition assays using $10 \mu \mathrm{g} / \mathrm{ml}$ MnPs A, C, Y, and W-135 as the competitor. We examined the effect of inhibition on binding of reference sera CDC 1992 and 89SF-2 in a multi-plex format and, in the majority of instances, determined that inhibition of 


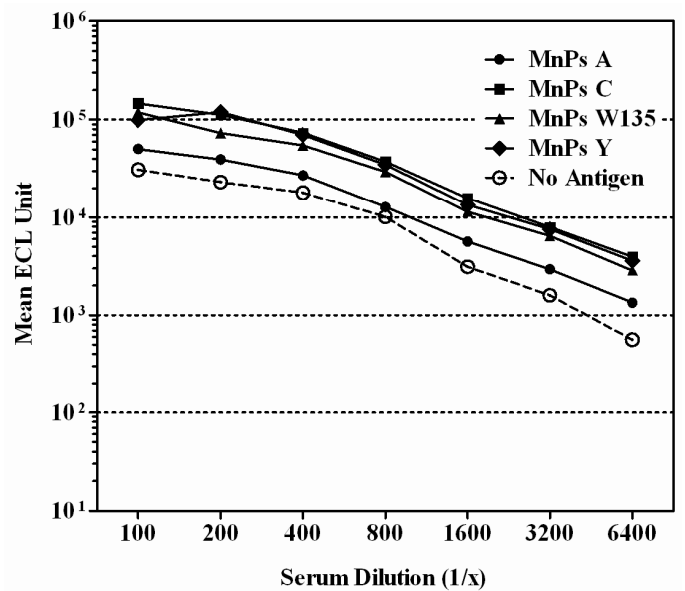

(a)

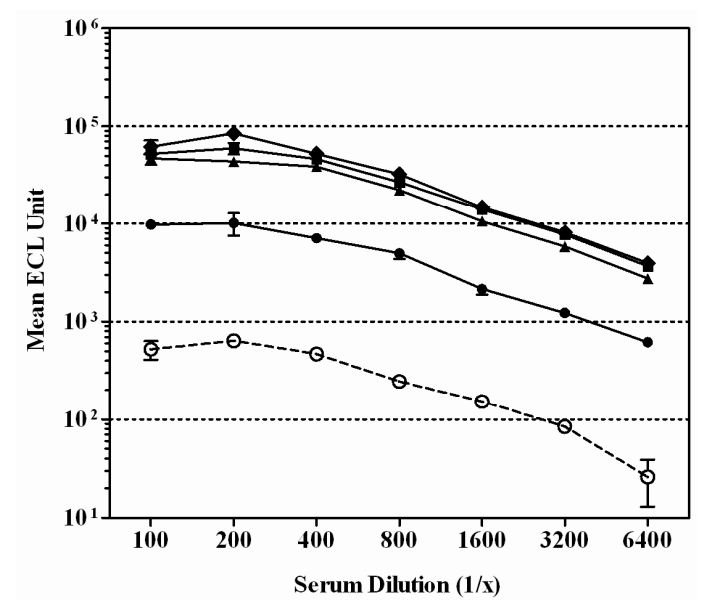

(c)

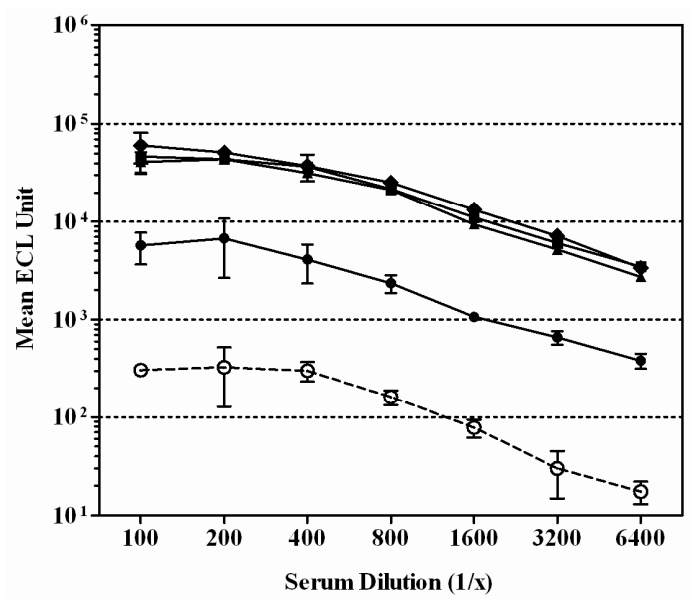

(e)

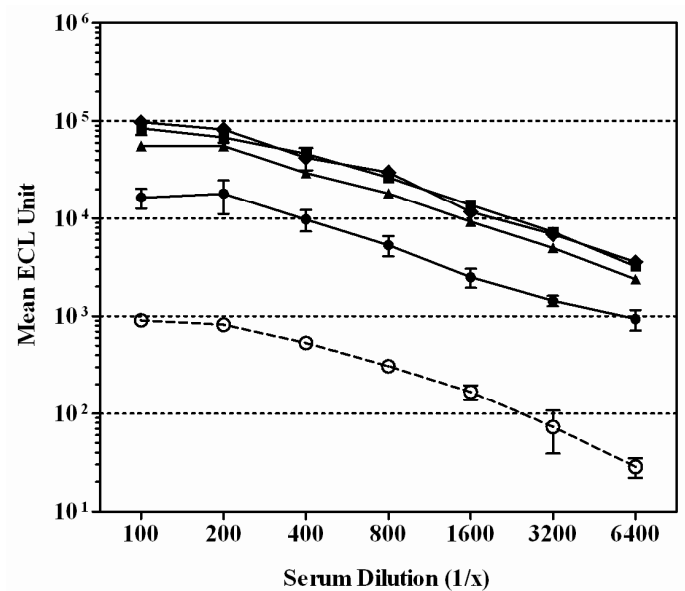

(b)

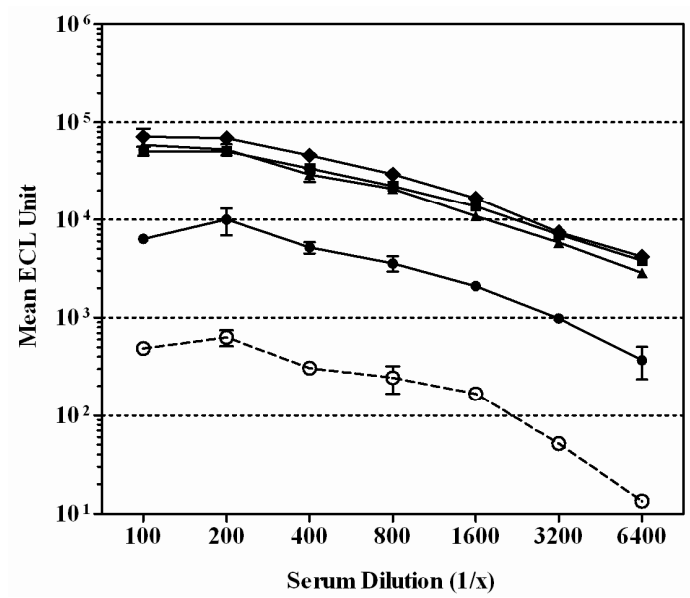

(d)

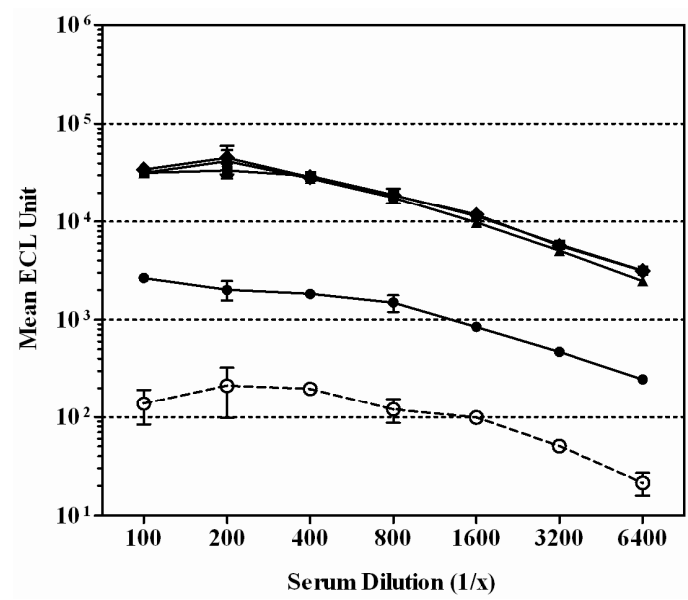

(f)

Figure 1. Effect of serum diluent buffers on reducing non-specific binding in single-plex ECL assay. CDC 1992 reference serum was serially diluted in six serum diluent buffers and tested on Multi-array ${ }^{\circledR}$ plates coated with MnPs A/C/Y/W-135 or un-coated. Data is represented as the mean \pm SEM of the ECL signal. The level of non-specific signal was significantly lower than the level of MnPs specific signals with the addition of $1 \%$ Blocker $B \pm N$ NaCl to PBS $(P<0.001)$. (a) Serum diluent buffer = PBS; (b) Serum diluent buffer = PBS $-1 \%$ Blocker B; (c) Serum diluent buffer = PBS $-1 \%$ Blocker B $+50 \mathrm{mM} \mathrm{NaCl}$; (d) Serum diluent buffer $=$ PBS $-1 \%$ Blocker $B+100 \mathrm{mM} \mathrm{NaCl}$; (e) Serum diluent buffer $=$ PBS $-1 \%$ Blocker B $+250 \mathrm{mM}$ $\mathrm{NaCl}$; (f) Serum diluent buffer = PBS - 1\% Blocker B $500 \mathrm{mM} \mathrm{NaCl}$. 


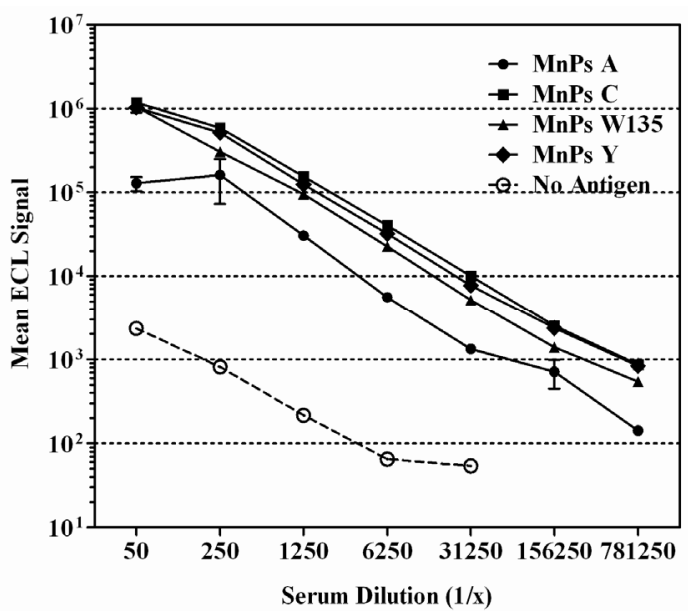

(a)

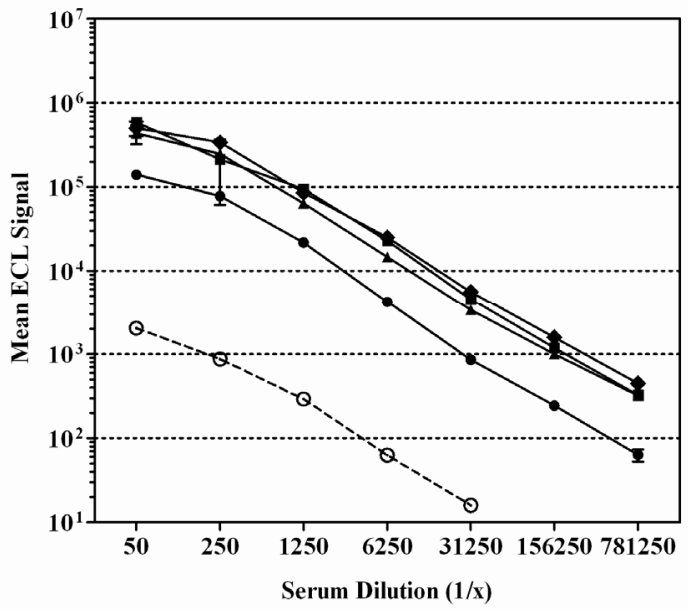

(b)

Figure 2. Effect of plate type on the signal level in a multiplex ECL assay. CDC 1992 reference sera were serially diluted on a (a) standard or (b) high binding Multi-plex ${ }^{\circledR}$ plates. Each spot in one well was coated with MnPs A/C/Y/W-135. Some wells were un-coated to show the level of serum nonspecific binding in this assay. Data is shown as the mean ECL signal \pm SEM.

the homologous serogroup polysaccharide was nearly complete (Table 1). For CDC 1992 and 89SF-2 reference sera, we observed $92 \%-98 \%$ and $77 \%$ - 99\% inhibition with the homologous serogroup polysaccharide, respectively. In some instances, however, some cross-reactivity between serogroups $\mathrm{A}$ and $\mathrm{C}$ or $\mathrm{Y}$ and $\mathrm{W}-135$ were observed for both reference sera. However, in each case the highest percent inhibition was observed with each homologous MnPs, indicating specificity of the assay.

\subsection{Meningococcal Vaccine Antibody Responses}

Anti-MnPs IgG responses from infant rhesus monkeys (IRM) that had been vaccinated three times with a half-
Table 1. Competitive inhibition studies of multi-plex MnPs $A, C, Y$, and W-135 ECL assay using meningococcal reference sera.

\begin{tabular}{|c|c|c|c|c|}
\hline \multicolumn{5}{|c|}{ CDC 1992 Reference Serum (\% inhibition) } \\
\hline \multirow{2}{*}{$\begin{array}{l}\text { Antibody } \\
\text { Response }\end{array}$} & \multicolumn{4}{|c|}{ MnPs Inhibitor } \\
\hline & A & $\mathrm{C}$ & $\mathrm{Y}$ & W135 \\
\hline A & 92 & 17 & 0 & 0 \\
\hline $\mathrm{C}$ & 67 & 98 & 21 & 0 \\
\hline Y & 16 & 0 & 98 & 70 \\
\hline W135 & 11 & 15 & 38 & 98 \\
\hline \multicolumn{5}{|c|}{ 89SF-2 Reference Serum (\% inhibition) } \\
\hline \multirow{2}{*}{$\begin{array}{l}\text { Antibody } \\
\text { Response }\end{array}$} & \multicolumn{4}{|c|}{ MnPs Inhibitor } \\
\hline & A & $\mathrm{C}$ & $\mathrm{Y}$ & W135 \\
\hline A & 77 & 49 & 0 & 7 \\
\hline $\mathrm{C}$ & 0 & 99 & 0 & 0 \\
\hline $\mathrm{Y}$ & 0 & 0 & 99 & 66 \\
\hline W135 & 0 & 0 & 48 & 98 \\
\hline
\end{tabular}

human dose of Menomune ${ }^{\circledR}$ or Menactra ${ }^{\circledR}$ were measured using the multi-plex MnPs ECL assay (Figure 3). Since previous experiments (data not shown) had demonstrated cross recognition of rhesus antibodies by the anti-human secondary antibody, this secondary antibody was used to determine antibody responses to vaccinated monkeys. The antibody concentrations in each sample were calculated based on the standard curve generated with the CDC 1992 reference serum. All of the rhesus sera showed low levels of pre-immune anti-MnPs IgG antibody. Menomune $^{\circledR}$ induced a small increase in the IRM's IgG responses for all four serogroups consistent with the concept that infant primates do not respond well to T-independent antigens (Figure 3(a)). In contrast, a single dose of the conjugate vaccine, Menactra ${ }^{\circledR}$, induced a marked increase ( $>100$ fold) of anti-MnPs IgG antibody response for all four serogroups contained in the vaccine (Figure 3(b)). This increase in IgG response appeared to reach a maximum after the second vaccine dose.

\section{Discussion}

Neisseria meningitidis infections remain a major public health issue worldwide. Several meningococcal vaccines have been licensed, with the most recent consisting of polysaccharide conjugated to protein carriers. Just last year, another meningococcal polysaccharide conjugate vaccine was licensed in the United States, Menveo ${ }^{\circledR}$ (Novartis) [20]. Sera from immunized individuals are evaluated in serum bactericidal assays as well as ELISAs to assess antibody function and polysaccharide immunogenicity. As vaccines become more complex, it is imperative that assays follow suit. In general, high throughput multiplex assays offer the benefits of speed, lower 


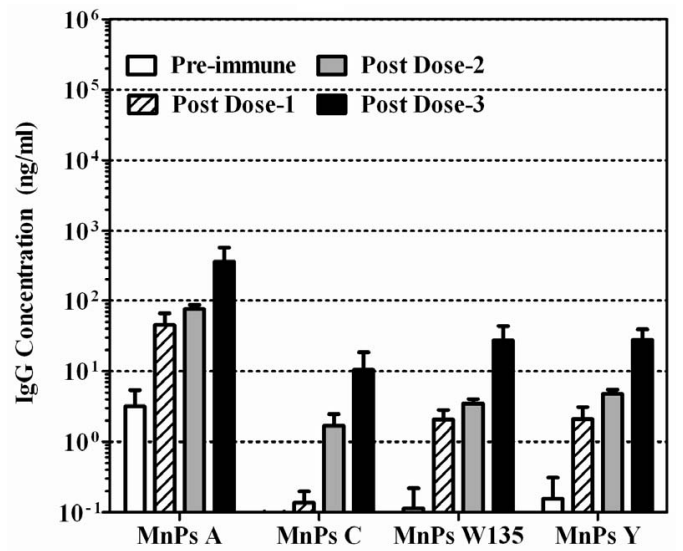

(a)

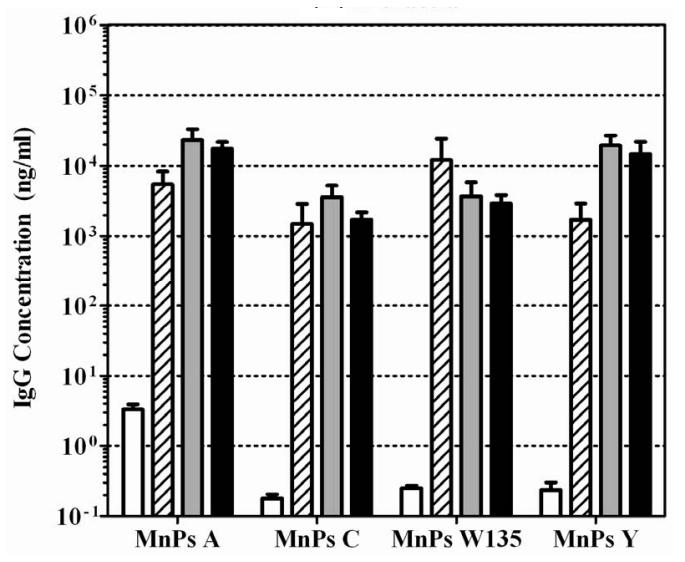

(b)

Figure 3. Anti-MnPs IgG responses from infant rhesus monkeys vaccinated with (a) Menomune ${ }^{\circledR}$ or (b) Menactra ${ }^{\circledR}$. Preimmune, post dose-1, post dose-2, and post dose- 3 serum samples were tested in four-plex ECL. CDC 1992 reference serum was the standard in the assay for determining IgG concentrations to all four MnPs. Data is shown as geometric mean concentration \pm SEM.

serum requirements, and reduced operator input. Adding to that, electrochemiluminescent (ECL) assays offer increased sensitivity, reproducibility, and lower sample volume compared to ELISAs.

In order to determine antibody responses for meningococcal vaccines, we developed a MnPs ECL assay. This assay uses a 96-well plate with all four MnPs (A, C, $\mathrm{Y}$, and W-135) passively adsorbed to the carbon surface within a single well. The 96-well ECL assay uses proprietary Multi-Array ${ }^{\circledR}$ or Multi-Spot ${ }^{\circledR}$ microplates with integrated carbon electrodes and a Sulfo-tag labeled secondary antibody that emits light when electrochemically stimulated. This signal is enhanced by the presence of a co-reactant (Tripropylamine/TPA) in the read buffer that is also stimulated when in proximity to the electrodes [21]. To best evaluate and optimize the assay, we used standard reference sera $[15,17]$ as well as evaluating se- rum antibody responses to licensed vaccines in infant rhesus monkeys, a clinically relevant animal model for human infant vaccination. Both CDC 1992 and 89SF reference sera have been used to evaluate and standardize antibody based assays $[22,23]$.

Initial experiments on the single-plex high binding plate demonstrated that a coating concentration of 50 $\mathrm{ng} /$ well with $\mathrm{MnPs}$ is optimal. Increasing the coating concentration to $250 \mathrm{ng} / \mathrm{well}$ did not improve the signal level. Due to the smaller surface area of the spots in the four-plex assay versus the single-plex assay, a lower coating concentration was found to be optimal (10 ng $\mathrm{MnPs} / \mathrm{spot} / \mathrm{well}$ ). Reduced surface areas require lower Ps coating concentrations; for example, a previously optimized pneumococcal polysaccharide (PnPs) ECL assay uses $5 \mathrm{ng} \mathrm{PnPs} /$ spot on a ten-spot/well format [12]. Due to the nature and binding of the carbon ECL plate, it was also determined that the addition of mHSA was not required for MnPs A binding to the surface of the plate, which is the case for ELISA assay due to the poor adsorption of this polysaccharide to the polystyrene surface. Of the blocking buffers evaluated, $3 \%$ Blocker B (non-fat milk) produced the lowest level of background noise for the assay. Several serum diluent buffers (PBS, PBS $+1 \%$ Blocker $\mathrm{B} \pm \mathrm{NaCl}$ ) were also evaluated to reduce the background signals in the assay. Adding 1\% Blocker B to $\mathrm{PBS}$ was able to solve this issue. Increasing $\mathrm{NaCl}$ concentrations lowered the levels ECL signals for all four $\mathrm{MnPs}$, especially for MnPs A. Out of the four groups studied here (A, C, W135, and Y), MnPs A is the only group with a phosphodiester backbone in its structure [24]. For nucleic acids which also contain phosphodiester backbones, salt can induce conformational changes in the DNA polymer $[25,26]$. The effect of salt on nucleic acid confirmation change can be attributed to the presence of strongly hydrated ions being excluded from the vicinity of hydrophobic solutes, thus causing greater salting out of hydrophobic solutes [27]. Similarly, studies had shown that MnPs A exhibits conformational behavior dependent on the nature of its counterion, and sodium ions favored the folding of the MnPs A chain into a globular conformation [28]. This may also explain the reason why increasing concentration of $\mathrm{NaCl}$ resulted in decreased binding for MnPs A. Salting out of hydrophobic regions due to ion hydration alters MnPs A's conformation, which may change its binding kinetics. Since PBS has a physiologic ionic strength, the use of PBS $+1 \%$ Blocker $\mathrm{B}$ as a serum diluent buffer would be the most "physiologically relevant" and hence was chosen as the serum diluent buffer. The selection of this diluent allows for binding of low affinity antibodies that might not bind in the presence of higher concentrations of $\mathrm{NaCl}$.

MnPs specificity was evaluated by competitive inhibi- 
tion with homologous and heterologous serogroup MnPs. Inhibition assays with MnPs have been used to demonstrate antibody binding specificity for standard reference and post-vaccination sera [18]. When assessing both CDC1992 and 89SF-2 reference sera in competitive inhibition assays, we observed near complete (80\% - 99\%) inhibition for each homologous MnPs. When evaluating heterologous MnPs in the competitive inhibition assays, we observed varying degrees of cross-reactivity. This generally trended between MnPs A and C or between MnPs Y and W135. The cross-reactivity observed may be due to similarity in the MnPs structure and impurities leftover from the purification process. Both MnPs W135 and $\mathrm{Y}$ have two sugars in their structures [29]. MnPs Y contains equimolar amounts of D-glucose and N-acetylneuraminic acid and is partially O-acetylated MnPs W135 differs from that of MnPs Y only in the absence of Oacetylation and the presence of D-galactose in the place of D-glucose. In addition, since the purification process of MnPs had not been optimized, a measurable amount of lipooligosaccharide (LOS) is present in these MnPs preparations. LOS from Neisseria meningitidis is composed of inner cores of L-glycero-D-manno-heptose and 3-deoxy-D-manno-oct-2-ulosonic acid, and anchored in the outer membrane by lipid A [30]. These components along with other outer membrane antigens may contribute to the cross-reactivity seen in the assay. Despite the cross-reactivity observed, the multi-plex ECL assay demonstrated specificity for the homologous MnPs. This assay is sensitive, fast, and reproducible with lower sample volume requirements. A competitive inhibition assay should be done in the traditional single ELISA to determine if similar cross-reactivity is present and the result between the two assays should be compared.

After development of the meningococcal A, C, Y, W135 multiplex ECL assay, we then tested IRMs serum samples from meningococcal vaccine studies. In two separate studies, we vaccinated IRMs with either Menomune ${ }^{\circledR}$ or Menactra ${ }^{\circledR}$ and collected blood throughout the course of the study. Pre-immune and hyper-immune sera were tested in the newly developed assay to determine the IgG antibody concentrations using CDC 1992 reference serum as assay standard. Vaccination with Menomune $^{\circledR}$ or Menactra ${ }^{\circledR}$ increased IgG responses for all four MnPs serogroups. However, these responses were higher in Menactra ${ }^{\circledR}$ vaccinated IRMs than those vaccinated with Menomune ${ }^{\circledR}$.

It is well known that polysaccharide vaccines induce a $\mathrm{T}$-cell independent antibody response, predominantly IgM. Conjugation of the polysaccharide to a protein carrier changes this response to a T-cell dependent response and hence increases the immunogenicity of the vaccine. Like human infants, IRMs do not respond well to uncon- jugated polysaccharide. Our data supports this, as Menomune ${ }^{\circledR}$ immunized IRMs produced a much lower IgG response than Menactra ${ }^{\circledR}$ immunized infant rhesus monkeys. In a similar comparison, Keyserling et al. reported higher serum bactericidal antibody responses three years post vaccination when comparing Menactra $^{\circledR}$ versus Menomune $^{\circledR}$ vaccinated healthy adolescents in a human clinical trial [31]. Our immunogenicity data, which reveals these differences in the unconjugated and conjugated vaccines, supports the applicability of the multiplex meningococcal ECL assay for evaluation of investigational and licensed vaccines. The IgG concentration of MnPs in the CDC 1992 reference serum was originally determined in an ELISA assay. We used the same CDC 1992 reference serum as a standard for our multi-plex ECL assay, therefore we expect the results to be comparable with an ELISA based assay. For a full circle comparison, the vaccine immunized monkey samples should be tested in the gold-standard MnPs ELISA assay and compared to the IgG concentrations generated in the ECL assay.

In conclusion, we have successfully developed a multi-plex ECL assay to assess post immunization antibody responses with meningococcal $\mathrm{A}, \mathrm{C}, \mathrm{Y}$, and $\mathrm{W} 135$ vaccines. This methodology is both time and serum conservative compared to conventional meningococcal polysaccharide ELISAs. This assay can also be used for vaccine efficacy screening. We developed this assay on a four spots/well format in a 96-well plate; however it can be expanded to ten spots/well with the addition of other antigens. With this MSD technology we also have the capability to develop a four-plex 384-well plate assay. This would allow for evaluation of combination vaccines as well as enhanced meningococcal vaccines containing additional polysaccharides or conserved proteins.

\section{Acknowledgements}

We would like to thank the animal care and veterinary staff at Covance, Inc (Alice, TX) for performing the infant rhesus monkey studies. We also thank Craig Przysiecki for critical review of the manuscript. All authors are employees of Merck \& Co., Inc. and may potentially own stock and/or hold stock options in the company.

\section{REFERENCES}

[1] F. M. LaForce, et al., "The Meningitis Vaccine Project," Vaccine, Vol. 25, Supplement 1, 2007, pp. 97-100. doi:10.1016/j.vaccine.2007.04.049

[2] M. E. Pichichero, "Meningococcal Conjugate Vaccines," Expert Opinion on Biological Therapy, Vol. 5, No. 11, 2005, pp. 1475-89. doi:10.1517/14712598.5.11.1475

[3] M. Broker and S. Fantoni, "Meningococcal Disease: A 
Review on available Vaccines and Vaccines in Development," Minerva Medica, Vol. 98, No. 5, 2007, pp. 575 -589 .

[4] J. D. Campbell, et al., "Safety, Reactogenicity, and Immunogenicity of a Tetravalent Meningococcal Polysaccharide-Diphtheria Toxoid Conjugate Vaccine Given to Healthy Adults," The Journal of Infectious Diseases, Vol. 186, No. 12, 2002, pp. 1848-1851. doi:10.1086/345763

[5] B. Cooper, L. DeTora and J. Stoddard, "Menveo ${ }^{\circledR}$ : A Novel Quadrivalent Meningococcal CRM197 Conjugate Vaccine against Serogroups A, C, W-135 and Y," Expert Reviews of Vaccines, Vol. 10, No. 1, 2011, pp. 21-33. doi:10.1586/erv.10.147

[6] M. Rennels, et al., "Dosage Escalation, Safety and Immunogenicity Study of Four Dosages of a Tetravalent Meninogococcal Polysaccharide Diphtheria Toxoid Conjugate Vaccine in Infants," Pediatric Infectious Disease Journal, Vol. 23, No. 5, 2004, pp. 429-35. doi:10.1097/01.inf.0000126297.28952.f8

[7] M. D. Snape, et al., "Immunogenicity of a Tetravalent Meningococcal Glycoconjugate Vaccine in Infants: a Randomized Controlled Trial," The Journal of the American Medical Association, Vol. 299, No. 2, 2008, pp. 173-184. doi:10.1001/jama.2007.29-c

[8] G. F. Blackburn, et al., "Electrochemiluminescence Detection for Development of Immunoassays and DNA Probe Assays for Clinical Diagnostics," Clinical Chemistry, Vol. 37, No. 9, 1991, pp. 1534-1539.

[9] V. Guglielmo-Viret and P. Thullier, "Comparison of an Electrochemiluminescence Assay in Plate Format over a Colorimetric ELISA, for the Detection of Ricin B Chain (RCA-B)," Journal of Immunological Methods, Vol. 328, No. 1-2, 2007, pp. 70-78. doi:10.1016/j.jim.2007.08.003

[10] R. M. de Voer, et al., "Development of a FluorescentBead-Based Multiplex Immunoassay to Determine Immunoglobulin G Subclass Responses to Neisseria meningitidis Serogroup A and C Polysaccharides," Clinical and Vaccine Immunology, Vol. 15, No. 8, 2008, pp. 1188-93. doi:10.1128/CVI.00478-07

[11] T. B. Martins, et al., "Development of a Multiplexed Fluorescent Immunoassay for the Quantitation of Antibody Responses to Four Neisseria meningitidis Serogroups," Journal of Immunological Methods, Vol. 342, No. 1-2, 2009, pp. 98-105. doi:10.1016/j.jim.2008.12.003

[12] R. D. Marchese, et al., "Optimization and Validation of a Multiplex, Electrochemiluminescence-Based Detection Assay for the Quantitation of IgG Serotype-Specific Antipneumococcal Antibodies in Human Serum," Clinical and Vaccine Immunology, Vol. 16, No. 3, 2009, pp. 387-396. doi:10.1128/CVI.00415-08

[13] C. E. Frasch, "Production and Control of Neisseria meningitidis Vaccines," Advances in Biotechnological Processes, Vol. 13, 1990, pp. 123-145.

[14] A. L. Lee, M. S. Rienstra, W. E. Manger and R. D. Sitrin, "Process for Converting Lipid-Containing Bacterial Capsular Polysaccharide into Lipid-Free Polysaccharide," Merck \& Co., Rahway, 1994.

[15] P. K. Holder, et al., "Assignment of Neisseria meningitis- dis Serogroup A and C Class-Specific Anticapsular Antibody Concentrations to the New Standard Reference Serum CDC1992," Clinical and Diagnostic Laboratory Immunology, Vol. 2, No. 2, 1995, pp. 132-137.

[16] D. C. Phipps, et al., "Standardization of ELISA for the Quantitation of Antibodies to S. pneumoniae Capsular Polysaccharides (PnPs)," Pediatric Research, Vol. 27, 1990, p. 179A.

[17] S. A. Quataert, et al., "Assignment of Weight-Based Antibody Units to a Human Antipneumococcal Standard Reference Serum, Lot 89-S," Clinical and Diagnostic Laboratory Immunology, Vol. 2 No. 5, 1995, pp. 590597.

[18] G. M. Carlone, et al., "Multicenter Comparison of Levels of Antibody to the Neisseria meningitidis Group A Capsular Polysaccharide Measured by Using an EnzymeLinked Immunosorbent Assay," Journal of Clinical Microbiology, Vol. 30, No. 1, 1992, pp. 154-159.

[19] A. J. Pollard and M. C. J. Maiden, "Meningococcal Disease: Methods and Protocols," In: J. M. Walker, Ed., Methods in Molecular Medicine, Vol. 67, Humana Press, Totowa, 2001.

[20] Advisory Committee on Immunization Practices (ACIP), "Licensure of a Meningococcal Conjugate Vaccine (Menveo) and Guidance for Use," Morbidity and Mortality Weekly Report, Vol. 59, No. 9, 2010, p. 273.

[21] D. R. Deaver, “A New Non-Isotopic Detection System for Immunoassays," Nature, Vol. 377, No. 6551, 1995, pp. 758-760. doi:10.1038/377758a0

[22] H. Joseph, et al., "Assignment of Neisseria meningitidis Serogroups A, C, W135, and Y Anticapsular Total Immunoglobulin $\mathrm{G}$ (IgG), IgG1, and IgG2 Concentrations to Reference Sera," Clinical and Diagnostic Laboratory Immunology, Vol. 11, No. 1, 2004, pp. 1-5.

[23] R. D. Marchese, et al., "Enzyme-Linked Immunosorbent Assay for Measuring Antibodies to Pneumococcal Polysaccharides for the PNEUMOVAX 23 Vaccine: Assay Operating Characteristics and Correlation to the WHO International Assay," Clinical and Vaccine Immunology, Vol. 13, No. 8, 2006, pp. 905-912. doi:10.1128/CVI.00014-06

[24] D. R. Bundle, I. C. Smith and H. J. Jennings, "Determination of the Structure and Conformation of Bacterial Polysaccharides by Carbon 13 Nuclear Magnetic Resonance. Studies on the Group-Specific Antigens of Neisseria meningitidis Serogroups A and X," The Journal of Biological Chemistry, Vol. 249, No. 7, 1974, pp. 2275-2281.

[25] C. W. Chen and J. S. Cohen, "Salt- and Sequence-Dependence of the Secondary Structure of DNA in Solution by ${ }^{31}$ P-NMR Spectroscopy," Biopolymers, Vol. 22, No. 3, 1983, pp. 879-893. doi:10.1002/bip.360220310

[26] M. Vorlickova, J. Kypr and V. Sklenar, "Salt-Induced Conformational Transition of Poly[d(A-T)]-Poly[d(A-T)]," Journal of Molecular Biology, Vol. 166, No. 1, 1983, pp. 85-92. doi:10.1016/S0022-2836(83)80052-7

[27] A. Kalra, N. Tugcu, S. M. Cramer and S. Garde, "Salting-In and Salting-Out of Hydrophobic Solutes in Aqueous Salt Solutions," The Journal of Physical Chemistry B, 
Vol. 105, No. 27, 2001, pp. 6380-6386.

doi:10.1021/ip010568+

[28] M. Foschiatti, et al., "Conformational Studies of the Capsular Polysaccharide Produced by Neisseria meningitidis group A," Carbohydrate Research, Vol. 344, No. 7, 2009, pp. 940-943. doi:10.1016/j.carres.2009.02.027

[29] A. K. Bhattacharjee, et al., "Structural Determination of the Polysaccharide Antigens of Neisseria meningitidis Serogroups Y, W-135, and BO1," Canadian Journal of Biochemistry, Vol. 54, No. 1, 1976, pp. 1-8. doi:10.1139/o76-001
[30] C. M. John, M. Liu and G. A. Jarvis, "Profiles of Structural Heterogeneity in Native Lipooligosaccharides of Neisseria and Cytokine Induction," Journal of Lipid Research, Vol. 50, No. 3, 2009, pp. 424-38.

doi:10.1194/jlr.M800184-JLR200

[31] H. Keyserling, et al., "Safety, Immunogenicity, and Immune Memory of a Novel Meningococcal (Groups A, C, Y, and W-135) Polysaccharide Diphtheria Toxoid Conjugate vaccine (MCV-4) in Healthy Adolescents," Archives of Pediatrics \& Adolescent Medicine, Vol. 159, No. 10, 2005, pp. 907-913. doi:10.1001/archpedi.159.10.907 